\title{
Comment on "Four methods of locating the egocenter: A comparison of their predictive validities and reliabilities"
}

\author{
JUNE ADAM and LAYNA BATEMAN \\ University of Calgary, Calgary, Alberta T2N IN4, Canada
}

\begin{abstract}
Barbeito and Ono (1979) recently reported a psychometric evaluation of four methods of measuring the egocenter. Evaluations of this kind can be extended, with a view to obtaining independent estimates of reliability and validity coefficients. In this way, the advantage(s) of one type of measure over another can be more clearly delineated.
\end{abstract}

Psychometric methods are rarely used as research tools by the experimental psychologist, despite their obvious potential usefulness in supplementing the kind of information yielded by the traditional experimental methods. It is, therefore, encouraging to see the recent psychometric evaluation of four techniques designed to locate the "egocenter" in studies of visual direction (Barbeito \& Ono, 1979; Mitson, Ono, \& Barbeito, 1976). Barbeito and Ono (1979) recently reported both reliability coefficients and predictive validity coefficients for Fry's (1950), Funaishi's (1926), Howard and Templeton's (1966), and Roelofs' (1959) methods of locating the egocenter. The authors concluded that Howard and Templeton's method is to be preferred over the rest, cautiously suggesting that "its advantage over the other methods is one of greater precision (and possibly accuracy) rather than superior theoretical foundation" (Barbeito \& Ono, 1979, p. 34). The tentative nature of Barbeito and Ono's conclusions derives partly from their recognition that the relative magnitudes of the predictive validity coefficients reflected differences in the reliabilities of the methods, as well as differences in the intrinsic relationships between the four measures of egocenter and three visual direction criterion tasks. The purpose of this paper is to point out ways in which Barbeito and Ono's psychometric evaluation can be extended, with a view to obtaining independent estimations of reliability and validity coefficients, and hence stronger evidence regarding the reason(s) for the superiority of one method over another.

A correlation between two variables will always be attenuated by lack of precision in measuring those variables; thus, Barbeito and Ono's (1979) validity coefficients to a greater or less extent reflect unreliability of the observed criterion measures and unreliability of the four methods of measuring the two egocenter dimensions (the latter determined the predicted criterion measures, which were then correlated with the observed values). This paper suggests the possibility of estimating the intrinsic relationship between the predicted and observed criterion scores by applying a for- mula that is analogous to the "correction for attenuation" commonly used in the field of psychometrics (e.g., Nunnally, 1967). This formula permits one to estimate how much a correlation would be raised (1) if the observed criterion measures were perfectly reliable, (2) if the predicted criterion measures were perfectly reliable, or (3) if both were perfectly reliable. In this way it is possible to estimate validity coefficients that are uncontaminated by imprecision of measurement for either or both of the variables. Formulas for Conditions 1,2 , and 3 are shown below.

$$
\begin{aligned}
& r_{x T_{c}}=r_{x c} / \sqrt{r_{c c^{1}}}, \\
& \mathrm{r}_{\mathrm{T}_{\mathrm{x}} \mathrm{c}}=\mathrm{r}_{\mathrm{Xc}} / \sqrt{ } \mathrm{r}_{\mathrm{xx}^{1}}, \\
& { }^{T_{X} T_{c}}=r_{x c} /\left(\sqrt{x_{x X^{1}}} \sqrt{ } \mathrm{r}_{c c^{1}}\right),
\end{aligned}
$$

where $r_{x c}=$ obtained correlation coefficient between predicted criterion scores $(\mathrm{x})$ and obtained criterion scores (c), $\mathrm{r}_{\mathrm{xT}_{\mathrm{c}}}=$ estimated correlation coefficient between predicted criterion scores and "true" criterion scores, ${ }^{\mathrm{r}} \mathbf{T}_{\mathbf{x}} \mathrm{c}=$ estimated correlation coefficient between "true" predicted criterion scores (i.e., the predicted criterion scores for perfectly reliable underlying measures) and the obtained criterion scores, $r_{\mathrm{cc}^{1}}=$ reliability coefficient for obtained criterion scores, and $r_{\mathbf{x x}^{1}}=$ reliability coefficient for predicted criterion scores.

For Barbeito and Ono's (1979) purposes, namely, the comparison of validity coefficients for the four methods of locating the egocenter, little would be gained by correcting the coefficients for unreliability of the obtained criterion measures. Lack of precision in measuring a particular criterion must affect all coefficients alike, decreasing them by a factor of ${\sqrt{\mathrm{rcc}}}$. Quite obviously, however, lack of precision in the particular method of measuring the egocenter must differentially affect the coefficients unless the reliability coefficients for the methods are equal. If the validity coefficients are to reflect solely the extent to which the different egocenter measures are potentially 
capable of predicting the observed criterion scores, they must be converted to estimates of the correlation between obtained measures on the criterion and "true" predicted criterion scores (i.e., the predicted criterion scores for a perfectly reliable underlying measure). This in turn must entail the assessment of a reliability coefficient for the predicted values. Barbeito and Ono (1979) provide reliability coefficients for the egocenter dimension measures on which the predicted values are based. However, other aspects of their study preclude an analysis of the kind proposed here. We suggest a modification of Barbeito and Ono's approach to the problem. In so doing, one purpose is to underline the usefulness of the general approach employed by the earlier authors.

When validity coefficients are to be "corrected" for unreliability of the test (the analogue of Barbeito and Ono's, 1979, criterion scores as predicted from the two egocenter dimension measures), it is extremely important to obtain a sound estimate of test reliability and to ensure that the error of measurement that affects the reliability of the test is the same error of measurement that affects the correlation between the test and the criterion. From the first point, it follows that reliability estimates should be based on large samples of individuals (Nunnally, 1967) and that validity coefficients should be based upon the same samples, so that the two coefficients are matched with regard to the variability of the scores (McNemar, 1969). On the second point, when both test and criterion scores are collected in a single testing session, the appropriate measure of reliability is one of the internal consistency methods. If the test and criterion scores are collected in separate sessions, then reliability should be estimated by the testretest method, with the time interval matching that between the test and criterion scores (Allen \& Yen, 1979).

The above conditions are not easy to meet. Indeed, it is impossible to satisfy all of them unless (1) both the test and criterion measures can be obtained in a single session, so that the necessary judgments for the two types of measures are alternated or randomly ordered within one series of trials, or (2) the criterion measures are obtained in a first series of trials, the test measures in a second series administered at another time, and the retest measures in a third, with intersession time intervals equal. The first alternative obviously poses problems regarding changes of apparatus and changes in the instructions given to individuals, apart from the length of time required to collect the measures. For similar reasons it is assumed that in the latter alternative, the different test and criterion tasks are given in succession within a series of trials. The latter possibility for Barbeito and Ono (1979) requires only a simple rearrangement of the experiment as it was originally conducted, namely, an interchange of the procedures on two testing occasions. In the Barbeito and Ono experiment, the test-retest time interval for measures of the egocenter was twice the interval between the measurement of the egocenter and measurement of the criterion, so that errors of measurement for the reliability and validity estimates were based on different time spans. In the procedure suggested here, the test-retest interval for the egocenter equals the interval between test and criterion, so that errors of measurement are equated for time span. From the first two sessions, it is then possible to compute the validity coefficients of the four methods for the three visual direction tasks. The data from the second and third sessions can then be analyzed specifically for the test-retest reliability coefficients pertaining to the four methods of measuring the egocenter. In addition, by computing predicted criterion scores separately for the last two sessions, it is possible to obtain reliability coefficients for the predicted criterion scores used in the validity coefficients. The validity coefficients can subsequently be corrected for unreliability of the predicted criterion scores (in effect, for unreliability of the method of measuring the egocenter), so that comparisons of these coefficients reflect only the relative amounts of criterion variance explained by the four measures of egocenter. In this way the advantage of one type of measure over another can be described in terms of reliability and validity as quite separate properties.

Not enough emphasis can be placed on the need for sound reliability estimates if the correction for attenuation formula is to be applied. As a further caution, it should be stressed that a method of measurement is only as useful as it is reliable; the corrected validity coefficient in no way "improves" the method for practical uses. However, if properly applied, the correction for attenuation can identify the method that has the greatest potential value as a predictive or explanatory device. This in turn identifies to researchers the method on which efforts should be concentrated for the improvement of reliability.

\section{REFERENCES}

Allen, M. J., \& YEN, W. M. Introduction to measurement theory. Monterey, Calif: Brooks/Cole, 1979.

Barbeito, R. A., \& ONo, H. Four methods of locating the egocenter: A comparison of their predictive validities and reliabilities. Behavior Research Methods \& Instrumentation, 1979, 11, 31-36.

FRY, G. A. Visual perception of space. American Journal of Optometry, 1950, 27, 531-553.

Funaishi, S. Weiteres über das Zentrum der Sehrichtungen. Albrecht von Graefes Archiv für Ophthalmologie, 1926, 117, 296-303.

Howard, I. P., \& Templeton, W. B. Human spatial orientation. New York: Wiley, 1966.

McNemar, Q. Psychological statistics. New York: Wiley, 1969.

Mitson, G. L., Ono, H., \& Barbeito, R. Three methods of measuring the location of the egocenter: Their reliability, comparative locations and intercorrelations. Canadian Journal of Psychology, 1976, 30, 1-8.

Nunnally, J. Psychometric theory. New York: McGraw-Hill, 1967.

Rozlofs, C. O. Considerations on the visual egocentre. Acta Psychologica, 1959, 16, 226-234.

(Received for publication October 31, 1979; revision accepted January 23,1980 .) 